\title{
RDH10 and ATRA sensitize triple-negative breast cancer to taxane-based chemotherapy through regulation of PIN1
}

Jordan Grainger

Mayo Clinic Graduate School for Biomedical Sciences

Lingxin Zhang

Mayo Clinic College of Medicine and Science

Jia Yu

Mayo Clinic College of Medicine and Science

Peter T Vedell

Mayo Clinic College of Medicine and Science

Kevin Thompson

Mayo Clinic College of Medicine and Science

Krishna R Kalari

Mayo Clinic College of Medicine and Science

Vera J Suman

Mayo Clinic College of Medicine and Science

Judy C Boughey

Mayo Clinic College of Medicine and Science

Matthew P Goetz

Mayo Clinic College of Medicine and Science

Liewei Wang ( $\nabla$ wang.liewei@mayo.edu )

Mayo Clinic College of Medicine and Science

\section{Research}

Keywords: TNBC, biomarkers, chemotherapy-response, RDH10, PIN1, breast, BEAUTY, ATRA

Posted Date: October 7th, 2020

DOI: https://doi.org/10.21203/rs.3.rs-86064/v1

License: (c) (1) This work is licensed under a Creative Commons Attribution 4.0 International License.

Read Full License 


\section{Abstract}

Background: With few targeted therapies available for triple-negative breast cancer (TNBC), there is a critical need to identify biomarkers that can predict chemotherapy-response. Copy number amplifications (CNAmps) have been implicated in tumorigenesis, progression, and treatment response. Previously, we performed a prospective neoadjuvant chemotherapy study (BEAUTY) and identified a $5 \mathrm{Mb}$ CNAmp in TNBC patients associated with pathological complete response rates to taxane- and anthracycline-based chemotherapy. Here we further interrogated this amplicon and its role in chemotherapy response.

Methods: Using siRNA screening of genes identified in the $5 \mathrm{Mb}$ CNAmp, followed by cytotoxicity assays, we identified RDH10 significantly associated with chemo-sensitivity in TNBC cell lines. Functional studies were performed using RDH10 knockdown or overexpression followed by cytotoxicity, cell proliferation, luciferase reporter assays, HPLC or western blot.

Results: RDH10 is a rate-limiting step involved in the synthesis of all-trans retinoic acid (ATRA) - the active metabolite of retinoid metabolism that is frequently aberrant in cancer. Previous studies demonstrated ATRA to be a potent inhibitor of PIN1, a key regulator of several oncogenic signaling pathways often amplified in TNBC. We discovered that genetic manipulation of RDH10 affected ATRA intracellular concentrations, contributing to PIN1 degradation and taxane-response. Furthermore, co-treatment of TNBC cells with ATRA significantly increased taxane-sensitivity.

Conclusion: RDH10 amplification increases endogenous levels of ATRA and degrades PIN1 in TNBC. ATRA-mediated degradation of PIN1 sensitizes TNBC to chemotherapy, suggesting that RDH10 may be a novel biomarker of taxane response and the addition of ATRA to standard chemotherapy may improve chemo-sensitivity.

\section{Background}

Breast cancer is the most common cancer in women, and second leading cause of death among women in the United States (1). Triple negative breast cancer (TNBC) accounts for 15-20\% of breast cancer cases and is marked by the lack of human epidermal growth factor receptor 2 (HER2), estrogen receptor (ER), and progesterone receptor (PR) via immunohistochemistry (2). Unlike other subtypes of breast cancer, TNBC is the most aggressive subtype and has the worst prognosis (3). With a paucity of targeted therapies available for TNBC, cytotoxic agents including taxane and anthracycline/cyclophosphamide chemotherapy remain the backbone of (neo) adjuvant therapies. Although complete responses to neoadjuvant treatment (pCR) are associated with high cure rates, only $30-50 \%$ of patients achieve a pCR with standard anthracycline and taxane based therapies (4). Therefore, there is a great need to identify biomarkers and additional therapies to overcome chemo-resistance in TNBC.

With advances in next-generation sequencing (NGS) and expression profiling, TNBC is now known to be a disease containing a collection of genomic aberrations that contribute to tumorigenesis, tumor progression and treatment response (5). Copy number alterations (CNAs) are structural variants able to 
affect a wider region of the cancer genome than any other type of genetic aberration, causing the concomitant deletion or amplification of many different genes; therefore, much interest has been directed towards characterizing them (6). Of the 21,000 CNAs identified in various cancers, many are found to overlap, generating putative CNA "hotspots" (7). Some "hotspots" have been reported to be strongly associated with clinical phenotypes resulting in the discovery of novel therapeutic options for many cancer types (8-10). While these regions encompass canonical oncogenes (e.g., MYC) or tumor suppressors (e.g., TP53, ATM, CDKN2A/B), many other genes within these regions may play a role in disease phenotype, such as drug response (11). Studying genes within CNAs identified from TNBC patient tumors may lead to the identification of novel treatment response biomarker(s) and provide the opportunity for therapeutic interventions for tumors resistant to standard chemotherapy.

The Breast Cancer Genome-Guided Therapy Study (BEAUTY) is a prospective neoadjuvant clinical study with a 132 patient cohort of women diagnosed with Stage I-III breast cancer (12). The objective of this study is to identify genomic alterations and perturbed pathways associated with patient response or resistance to chemotherapy. Therefore, Whole Exome Sequencing (WES) and RNA sequencing (RNAseq) were performed on patient tumor and germline biopsies at baseline, during, and following completion of neoadjuvant chemotherapy. Out of this cohort $(n=132)$, approximately one-third $(n=44)$ were diagnosed with TNBC. These patients received 12 weeks of taxane treatment, followed by 4 cycles of anthracycline/cyclophosphamide. Following surgery, patients $(n=41)$ were stratified based on pathological complete response ( $\mathrm{pCR}$ ) as either responders (achieving $\mathrm{pCR} ; \mathrm{n}=22$ ) or non-responders (non-pCR; $n=19$ ) to the treatment. Association studies with outcomes were then performed using the sequencing data. Previously, we determined that $31.8 \%(n=7)$ of TNBC responders shared a 5 Mb copy number amplification (CNAmp) on chromosome 8q, containing 29 genes (12). Here we present a potential novel biomarker, Retinol Dehydrogenase $10(\mathrm{RDH} 10)$ associated with response to paclitaxel in TNBC. Based on our understanding of RDH10 involvement in chemo-response, we also present a potential new therapeutic approach to increase sensitivity to taxane treatment in TNBC.

\section{Methods}

Cell culture and reagents. Cell lines BT549 (HTB-122), MDA-MB-231 (HTB-26), Hs578t (HTB-126), and HEK-293t (CRL-3216) were all purchased from ATCC. HEK-293t and Hs578t were cultured in Dulbecco's modified Eagle's medium (DMEM), BT549 in RPMI-1640 and MDA-MB-231 in Leibovitz (L-15) Media. All media were supplemented with $10 \%$ fetal bovine serum (FBS), 1\% Penicillin/Streptomycin, and $10 \mathrm{uM}$ retinol dissolved in absolute $\mathrm{EtOH}$ and cultured at $37 \rrbracket \mathrm{C}$ in a humidified incubator containing $5 \% \mathrm{CO} 2$. siRNAs were purchased from Horizon Discovery. Paclitaxel (PTX), epirubicin (Epi), retinol (ROL) and alltrans retinoic acid (ATRA) were purchased from Sigma. Antibodies against various proteins were as follows: PIN1 (Catalog \#: 10495-1-AP) (1:500) and RDH10 (Catalog \#: 14644-1-AP) (1:500) from ProteinTech, Vinculin (Catalog \#: V9264) (1:10,000) from Sigma-Aldrich, and Beta-Actin (Catalog \#: 4967) (1:1000) from Cell Signaling. 
RNA interference. For siRNA transfection, cells were forward transfected with the indicated siGENOME SMARTpool siRNA (Horizon Discovery) using Lipofectamine2000 (Life Technologies Invitrogen) for BT549 and Hs578t or TransfeX (ATCC) for MDA-MB-231 transfection reagents following the manufacturer's protocol.

mRNA quantification. RNA was purified using the Zymo Research Quick-RNA MiniPrep Kit (Genesee Scientific). For each reaction, $100 \mathrm{ng}$ of total RNA was used for amplification of each target gene. mRNA levels were quantified by qRT-PCR using Power SYBR Green RNA-to- $\mathrm{C}_{\mathrm{T}}$ 1-Step Kit (Applied Biosystems by Thermo Fisher Scientific) and PrimeTime (IDT) pre-designed qPCR Primers. Gene expression analyses were performed using the $2^{-\triangle \Delta C t}$ method and GAPDH was used as the internal reference gene. Three independent experiments were performed.

Cytotoxicity and cell proliferation assay. For siRNA knockdown or overexpression studies, $1 \times 10^{5}$ and $2 \times$ $10^{5}$ cells/ well of BT549 and MDA-MB-231, respectively, were seeded in 6 well plates to achieve $80-90 \%$ confluence. The following day, cells were transfected with plasmids or siRNA using Lipofectamine2000 (for BT549) or TransfeX (for MDA-MB-231). Twenty-four hours after transfection, cells were trypsonized and reseeded at a cell density of 1000 cells/90 uL/well (for BT549, Hs578t) or 2000 cells/90 uL/well (for MDA-MB-231) in 96 well plates in triplicate. $10 \mathrm{uL}$ of increasing concentrations of paclitaxel or epirubicin were added to wells the following day. For ATRA treatment studies, a small amount of ATRA was added to a $1 \mathrm{~mL}$ aliquot of $100 \% \mathrm{EtOH}$ in the dark and vortexed until dissolved to achieve a final concentration $\sim 2 \mathrm{mM}$. The absorbance of ATRA was measured at $350 \mathrm{~nm}\left(\mathrm{e}=45,300 \mathrm{M}^{-1} \mathrm{~cm}^{-1}\right)$ using Thermo NanoDrop3000 Spectrophotometer and the Beer-Lambert Law was applied to calculate drug concentration. Fresh stock of ATRA was created prior to media changes. ATRA at indicated concentrations were then added to BT549 and MDA-MB-231 when seeding in 96 well flat-bottomed plates in triplicate. The following day, cells were treated with increasing concentrations of paclitaxel or epirubicin for 72 hours. For assessing cytotoxicity or proliferation, 10 uL Cyquant was added to the wells after appropriate drug treatment time and absorbance was measured using TECAN plate reader with excitation at $485 \mathrm{~nm}$ and emission detection at $520 \mathrm{~nm}$. Results were normalized to DMSO control and plots were generated using the GraphPad PRISM software. For cell proliferation assay, 10 uL Cyquant was added to wells every 24 hours and absorbance was measured as described above. Results were normalized to day 1 and were generated using GraphPad PRISM software.

Western blot analysis. Cell lines were lysed in CelLytic M (Sigma-Aldrich) lysis buffer containing phosphatase and proteasome cocktail inhibitors according to manufacturer's instructions. PDX tumors were mixed with NETN lysis buffer $(100 \mathrm{mM} \mathrm{NaCl}, 20 \mathrm{mM}$ Tris $\cdot \mathrm{HCl} \mathrm{pH}=0.8,0.5 \mathrm{mM}$ EDTA, NP-40) containing inhibitors and $0.5 \mathrm{~mm}$ RNase-free zirconium oxide beads in micro-centrifuge tubes and placed into a bullet blender for 10 minutes at $4 \mathbb{\complement C}$. Lysates were then normalized via Bradford assay and mixed with Tris-Tricine SDS sample buffer containing 2\% 2-ME and boiled for 10 minutes. Equal amounts of protein were subjected to electrophoresis in TGX Tris-Tricine SDS gels and were transferred to PVDF membranes. Membranes were blocked in TBST (1X TBS with 0.1\% Tween-20) with $5 \%$ non-fat milk 
powder for one hour. Membranes were washed with TBST and then incubated overnight at $4 \llbracket \mathrm{C}$ with indicated primary antibodies, followed by anti-mouse IgG or anti-rabbit IgG secondary antibody (1:2000) for one hour at room temperature. All blots were visualized with Supersignal WestPico or Supersignal West-Dura chemiluminescent ECL kits (Thermo Fisher) and image was documented by the Gel documentation system (Biorad).

Generation of stable cell lines. To establish stable cell lines, cells were infected by lentivirus. RDH10 shRNA clones V2LHS_267926 and V2LHS_100112 (Open Biosystems) and PIN1 shRNA clones NM_006221.x-616s1c1 and NM_006221.x-420s1c1 (Sigma-Aldrich) were utilized in this paper. Virus was generated in HEK-293T using the PMD2G and psPAX2 viral packaging vectors. Supernatant concentrated using the Takara Lenti-X Concentrator were then added to MDA-MB-231, BT549 and Hs578t cell lines with $8 \mathrm{ug} / \mathrm{mL}$ polybrene for 72 hours followed by puromycin selection for 2 weeks. Individual clones were then isolated and knockdown efficiency was verified by western blot.

Generation of overexpression constructs. Human RDH10 cDNA (Gene ID: 157506) was purchased from Horizon Discovery. cDNA was amplified by PCR and sub-cloned into pENTR shuttle vector using pENTR/D-TOPO cloning kit (Thermo Fisher). pENTR-RDH10 construct was subcloned into pcDNA3.2 destination vector using the Gateway LR Clonase kit (Invitrogen). Human Myc-DDK tagged PIN1 (CAT\#: RC202543) was purchased from Origene. QuickChange II XL Site-Directed Mutagenesis kit (Agilent) was used to generate S71D PIN1 construct. Inserts of all constructs were verified by Sanger sequencing. Plasmids were transiently transfected into BT549 using Lipofectamine 2000 and MDA-MB-231 using the TransfeX transfection reagents.

Intracellular all-trans retinoic acid concentration analysis. Cell lysates were separated and detected using high-performance liquid chromatography (HPLC)-UV method based on Williams et al. (13) with modifications. Specifically, cells were harvested, washed twice with PBS, and sonicated in $200 \mu \mathrm{L}$ of $0.25 \mathrm{M}$ ammonium acetate buffer pH4.0. $330 \mathrm{uL}$ acetonitrile was then added to samples and centrifuged at $14,000 \mathrm{rpm}$ for $10 \mathrm{~min}$ to remove cellular debris. The supernatant was filtered by $0.22 \mathrm{uM}$ nylon membranes. Samples were separated on a Shimadzu HPLC System (Shimadzu Corporation, Kyoto, Japan), equipped with a CBM-20A controller and LC-20AD pump (Shimadzu Corporation, Kyoto, Japan). Chromatographic separation of all-trans retinoic acid was achieved on a LiChrosorb® RP 18 - 5 (Supelco, Bellefonte, PA) C18 reversed-phase column ( $250 \mathrm{~mm} \times 4.6 \mathrm{~mm}, 5 \mathrm{uM})$. The mobile phase consisted of acetonitrile: methanol: $2 \%$ ammonium acetate: glacial acetic acid (79:2:16:3, v/v at a flow rate of $1.0 \mathrm{~mL} / \mathrm{min}$ at $25^{\circ} \mathrm{C}$ coupled with a Phenomenex Security Guard ${ }^{\mathrm{Tm}} \mathrm{C} 18$ guard column $4 \times 3 \mathrm{~mm}$ (Phenomenex, Torrance, CA). The mobile phase was degassed as well as vacuum filtered through 0.22 um nylon membranes before use. The HPLC system was equipped with the diode array detector SPDM20A (Shimadzu Corporation, Kyoto, Japan). 100 uL injections were used to analyze all-trans retinoic acid by UV absorption at $350 \mathrm{~nm}$. Data were acquired and processed with LabSolutions software version 5.85 (Shimadzu Corporation, Kyoto, Japan). Peaks from samples were then identified and quantified by comparison with the all-trans retinoic acid standard (Sigma, St. Louis, MI). 
Luciferase reporter assay Cells were seeded in 6 well plates to achieve $80-90 \%$ confluence the following day. Cells were forward transfected with the pGL3-RARE-luciferase construct, a gift from T. Michael Underhill (Addgene plasmid \#13458; http://n2t.net/addgene: 13458; RRID: Addgene_13458) (14), and pRL-TK internal control plasmid in a 1000:1 ratio using Lipofectamine2000 (for BT549, Hs578t) or TransfeX (for MDA-MB-231). The following day, cells were trypsonized and reseeded at a cell density of 1000 cells/100 uL/well in 96 well plates in sextuplicate. 72 hours later, luciferase activity was detected using the Dual-Glo Luciferase Assay System (Promega) kit following the manufacturer's instructions.

KM-Plotter database Association between the mRNA expression levels of DAPK1 and Relapse-Free Survival (RFS) was analyzed using the online database, KM-plotter (kmplot.com) using the gene expression data and the survival information of patients with breast cancer using all available datasets (15). Cohorts of patients were split by median expression. For subtype specification, the restriction analysis were default ("all") with the following exceptions: ER + subtype: ER status- ER-positive; HER2 + subtype: HER2 status- HER2 positive; TNBC subtype: ER status- ER-negative, PR status- PR negative, HER2 status- HER2 negative.

Statistical analysis Statistical tests were performed using GraphPad Prism Software; All figures presented have been replicated by 3 independent experiments and are reported as the mean \pm SD from triplicate data of one experiment, followed by determining significant differences using the two-tailed student $t$-test where ${ }^{\star} P<0.01,{ }^{*} P<0.001$, and ${ }^{*} * * P<0.0001$ unless otherwise stated. The Kaplan-Meier survival plots with the number at risk, hazard ratio (HR) and log-rank p-values were obtained using the KM plotter website. Log-rank P-values $<0.05$ was used to indicate a statistically significant difference.

\section{Results}

\section{siRNA cytotoxicity screen reveals RDH10 association with taxane response in TNBC}

We previously identified a $5 \mathrm{Mb}$ copy-number amplification on chromosome 8q (chr8q13.1-.3 and chr8q21.11-.12) in TNBC tumors associated with pCR (12). This region contains 29 genes. We first removed any RNA-coding genes and genes without any known function, leaving 20 protein-coding genes (Fig. 1A). We next utilized RNA sequencing data available in TNBC cell lines to eliminate genes with low baseline expression using an RPKM threshold of 0.1 , which corresponds to a false-discovery rate (FDR) and false-negative rate of $5 \%(16-18)$, narrowing our list to 17 genes (Fig. $1 \mathrm{~A})$. Finally we eliminated any genes that had poor knockdown efficiency $(\leq 50 \%)$ in both TNBC cell lines BT549 and MDA-MB-231 as determined by qRT-PCR, leaving 10 genes for functional study. To identify predictive biomarkers of treatment response to chemotherapy, siRNA cytotoxicity screening was performed in TNBC cell lines, BT549 and MDA-MB-231, with taxane (paclitaxel) or anthracycline (epirubicin)- two classes of drugs used in the BEAUTY study (12) as part of the neoadjuvant chemotherapy regimen (Fig. 1B). Following this screening, four genes showed a more resistant phenotype to paclitaxel compared to control ( $p$-value < 0.05) in either BT549 or MDA-MB-231 cells (Fig. 1C, Supplementary Table 1). Only RDH10 showed a consistent phenotype in both cell lines. 


\section{RDH10 expression regulates cell proliferation, taxane sensitivity and intracellular ATRA in TNBC}

To study the role of RDH10 in chemo-response, we generated stable knockdowns in TNBC cell lines BT549 and Hs578t, using two individual shRNA constructs targeting RDH10 (Fig. 2A, B). In addition, we performed transient overexpression of RDH10 in BT549 and MDA-MB-231 (Fig. 2C, D). Hs578t was utilized for knockdown studies and MDA-MB-231 was utilized for overexpression studies as each had higher and lower endogenous levels of RDH10, respectively. We utilized these lines to determine TNBC response to paclitaxel and epirubicin. We found that loss of RDH10 only increased resistance to paclitaxel in both BT549 and Hs578t with no effect observed with epirubicin (Fig. 2A, B). Conversely, overexpression of RDH10 only increased sensitivity to paclitaxel, with no effect with epirubicin (Fig. 2C, D). We also determined that loss of RDH10 increased cell proliferation (Fig. 2A, B) while overexpression of RDH10 decreased cell proliferation (Fig. 2C, D).

$\mathrm{RDH} 10$ is the rate limiting step involved in the synthesis of all-trans retinoic acid (ATRA) - the active metabolite in retinoid metabolism (Fig. 3A). Since RDH10 is one of over $20 \mathrm{RDH}$ isoforms with known catalytic activity (19), we sought to determine the specific impact RDH10 had on intracellular ATRA concentrations. We utilized HPLC-UV detection to quantify the endogenous levels of ATRA in whole cell lysates. We found that following knockdown of RDH10 in BT549 and Hs578t, intracellular ATRA was reduced by $40-60 \%$ (Fig. 3B, C), while overexpressing RDH10 in BT549 and MDA-MB-231 resulted in a four-fold increase in intracellular ATRA (Fig. 3D, E). These results were further confirmed by determining the ATRA effect on gene transcription through retinoid receptor using a luciferase reporter assay in cells transfected with a luciferase reporter construct with an upstream retinoic acid response element (RAREluc) (14).

Endogenous ATRA levels can also be regulated through intracellular metabolism by auto-inducible cytochrome P450s (CYPs)(20). While CYP3A4, CYP2C8, CYP26A1 and CYP26B1 contribute to systemic ATRA clearance (21), CYP26A1 and CYP26B1 are also expressed in extrahepatic tissues and influence tissue-specific intracellular ATRA concentrations $(20,22,23)$. To determine if ATRA produced by RDH10 overexpression affected its metabolism, we quantified CYP26A1 and CYP26B1 in TNBC cell lines. We found only CYP26A1 and CYP26B1 to be expressed in BT549 and MDA-MB-231 cells, respectively. Interestingly, overexpression of RDH10 significantly down-regulated transcripts of CYP26A1 in BT549, with no observed change in CYP26B1 in MDA-MB-231 (Fig. 3F).

\section{PIN1 loss increases taxane sensitivity and decreases cell proliferation similar to RDH10 overexpression in TNBC}

ATRA was previously identified through a large-scale, small-molecule screen to potently bind to and cause the ablation of peptidyl-prolyl cis/trans isomerase, NIMA interacting-1 (PIN1) (24). PIN1 is often amplified in aggressive forms of cancer, such as TNBC, and is involved in the cis-trans isomerization of phosphorylated serine/threonine-proline residues- activating over 50 oncogenes and inhibiting more than 20 tumor suppressors (25). To further study the mechanism of the RDH10 effect on chemo response, we sought to test the role of PIN1 in this process. First, we knocked down PIN1 in two TNBC cell lines 
(Supplementary Fig. 1A), followed by treatment with increasing doses of paclitaxel or epirubicin. We found that PIN1 knockdown decreased cell proliferation (Supplementary Fig. 1C) and increased sensitivity of BT549 and MDA-MB-231 to paclitaxel, but not epirubicin (Supplementary Fig. 1B); a phenomenon that was very similar to that of RDH10 overexpression (Fig. 2C, D).

\section{RDH10 affects TNBC taxane response and cell proliferation through PIN1}

To determine if the ability of RDH10 to modulate intracellular ATRA concentration and response to chemotherapy is through PIN1, we determined endogenous PIN1 levels after knocking down RDH10. We found that when RDH10 was knocked down and intracellular ATRA levels were low (Fig. 3B, C), PIN1 levels increased in BT549 and Hs578t (Fig. 4A, B), while overexpression of RDH10 increased intracellular ATRA levels (Fig. 3D, E) and decreased PIN1 levels (Fig. 4C, D); consistent with previous findings that ATRA can degrade PIN1 (24). Furthermore, using TNBC PDX tumor lysates obtained from the BEAUTY patients with either copy number amplification on chromosome $8 \mathrm{q}$ or those without, we found that tumors with $8 q$ amplification exhibited higher levels of RDH10 and lower levels of PIN1 compared to those without 8q amplification (Fig. 4E).

To further determine if the RDH10 effect on cell proliferation and chemo-response is through PIN1 regulation, we performed a double knockdown of both RDH10 and PIN1. We would expect that loss of both would reverse the cell proliferation and response to paclitaxel-induced by single RDH10 knockdown, which was confirmed by our experiments in Supplementary Fig. 2.

To further determine whether PIN1 activity is required for this process, we generated PIN1 stable knockdown cell lines (Supplementary Fig. 3) and then introduced either flag-tagged wild-type PIN1 or catalytically inactive S71D PIN1 (26), followed by overexpressing RDH10 (Fig. 5). In contrast to WT PIN1, overexpression of the catalytic inactive S71D PIN1 cannot rescue cell proliferation decreased by endogenous PIN1 knockdown. Our results also showed that RDH10 only decreased wild-type PIN1 levels, leading to decreased cell proliferation and increased TNBC sensitivity to paclitaxel (Fig. 5; Supplementary Table 2). This suggests that the cell proliferative and cytotoxic effects of RDH10 act through regulation of PIN1 and the effect is also dependent on PIN1 activity.

\section{ATRA sensitizes TNBC to taxane chemotherapy via PIN1 loss.}

ATRA has previously been used in the clinic in combination with chemotherapy for the treatment of acutepromyelocytic leukemia (APL), with complete remission rates greater than $90 \%$ (27). To determine if ATRA would increase the chemo-sensitivity in TNBC, we first tested the effect of ATRA alone, and found no evidence for cytotoxic effects on BT549 or MDA-MB-231 until concentrations reached as high as $100 \mathrm{uM}$ (Fig. 6A). We next determined the effect of ATRA on PIN1 levels with the assumption that PIN1 level is a pharmacodynamic marker for ATRA drug effect. When treated with $1 \mathrm{uM}$ and $10 \mathrm{uM}$ ATRA in BT549 and MDA-MB-231, PIN1 levels decreased (Fig. 6B). We next performed HPLC on whole cell lysates of cells treated with $1 \mathrm{uM}$ and $10 \mathrm{uM}$ ATRA. We found with 1 uM ATRA treatment, BT549 exhibited three times greater endogenous levels of ATRA compared to MDA-MB-231. Previous reports have shown that epithelial cells treated with retinoid-receptor agonists, including ATRA, can upregulate gene expression of 
RDH10 (28). We observed RDH10 to also be upregulated in BT549 with a lesser effect in MDA-MB-231 (Fig. 6B), suggesting ATRA-induced RDH10 expression could contribute to the observed differences in intracellular ATRA concentrations and PIN1 levels with 1 uM ATRA treatment between cell lines. However, both cell lines achieved similar endogenous ATRA concentrations with 10 uM ATRA regardless of RDH10 expression. We also noted that 10uM ATRA treatment resulted in similar intracellular ATRA levels that were achieved when RDH10 was overexpressed in both cell lines (Fig. 3D, E).

To determine if ATRA treatment could increase sensitivity of TNBC to chemotherapy, we co-treated BT549 and MDA-MB-231 with various concentrations of ATRA and paclitaxel or epirubicin. As expected, addition of 1 or 10 uM ATRA had limited effect on epirubicin sensitivity (Fig. 6E); However, there was a dose dependent sensitization to paclitaxel and a decrease in cell proliferation, consistent with that of PIN1 knockdown (Supplementary Fig. 1C) or RDH10 overexpression (Fig. 2C, D).

\section{Analysis of patient MRI tumor response to taxanes predicts pathological complete response in patients with RDH10 amplification.}

MRI imaging and RDH10 expression associations were performed on the 41 TNBC patients in BEAUTY following both the taxane portion and after full neoadjuvant chemotherapy (NAC) (Supplementary Table 3). Following completion of the taxane portion of NAC, 6 (85.7\%) of the 7 patients with RDH10 amplification and $16(47.1 \%)$ of 34 patients with wild-type RDH10 had imaging evidence of tumor response. We observed a trend toward a higher tumor response rate after taxane portion of NAC among those with RDH10 amplified TNBC disease than those with RDH10 wildtype TNBC disease (Fisher's exact test $p=0.0994)$. However, all $7(100 \%)$ TNBC patients with RDH10 amplification following full course of NAC had no invasive disease found in the breast and nodes at surgery (pCR), compared to 15 (44.1\%) of 34 TNBC patients with wild-type RDH10. TNBC pCR rate after NAC was significantly higher in those with RDH10 amplification than those with wild-type RDH10 (Fisher's exact test $p=0.0098$ ).

\section{Discussion}

Aberrant retinoid metabolism has been suggested to be a key factor driving tumorigenesis (29). Compared to healthy surrounding tissues, dramatically decreased gene expression of retinoid metabolizing enzymes and transporters corresponding with significantly lower endogenous ATRA concentrations has been observed in non-small cell lung (30), ovarian (13), breast (31, 32), colon (33), and prostate cancers (34). However, few studies have demonstrated the role of aberrant retinoid metabolism in drug-response. Previously, Guo et al. showed that elevated expression levels of several genes involved in alcohol metabolism that also play a role in retinoid metabolism were associated with decreased mortality in gastric cancer patients receiving 5-FU-based chemotherapy (35). Our study demonstrates for the first time in TNBC cell lines that the rate-limiting enzyme in retinoid metabolism, RDH10, significantly affects the intracellular concentration of ATRA (Fig. 3), leading to altered cell proliferation and sensitivity to taxane chemotherapy (Fig. 2). These findings were further confirmed through the addition of ATRA to cell culture media (Fig. 6). Interestingly, Resetkova et al. revealed an 
association between gene expression of another retinoid metabolizing enzyme, ALDH1, in the tumor microenvironment of TNBC patients and outcome after chemotherapy treatment. This study noted that while ALDH1 gene expression in tumor cells was not associated with overall or disease-free survival in TNBC patients, high stromal ALDH1 expression was significantly associated with outcome (36). Since ATRA is inherently lipophilic, we hypothesize with our in-vitro findings (Fig. 6), that ATRA produced in the microenvironment could have diffused into the surrounding tumor cells, contributing to TNBC chemoresponse for these patients. Of course future work is needed to confirm this hypothesis.

ATRA, in combination with chemotherapy, has been shown in randomized trials to improve long term survival and is the current standard treatment in the clinic for acute promyelocytic leukemia (APL), with complete remission rates of $90 \%$ (27). While it is widely thought ATRA binds to and degrades the PMLRARa fusion protein in APL, affecting downstream targets Cyclin D1 and NF-kB, inducing cellular differentiation and treating APL, the use of a pan-RAR agonist failed to mimic ATRA's effects. Work conducted by Wei et al. identified a novel non-canonical target for ATRA- PIN1 (24). PIN1 is highly amplified in aggressive forms of cancer, such as TNBC, and is involved in the cis-trans isomerization of phosphorylated serine/threonine-proline residues- activating over 50 oncogenes and inhibiting more than 20 tumor suppressors (25). PIN1's ability to bind to and isomerize these proteins can affect their structure and function, acting as an "on-off" switch for their activity. Low levels of PIN1 have been associated with favorable clinical outcome in many human cancers, including breast (37), and PIN1 has been suggested as an attractive non-toxic target to simultaneously block multiple cancer-driving pathways (38). While PIN1 ablation by ATRA has been shown to inhibit TNBC tumor growth in-vivo (24), its role in chemoresponse in TNBC has not been identified. Activation of NF-kB $(39,40)$, EGFR (41-43), Mcl-1 (44-46), AKT (47), Notch-1/3/4 (48-50) pathways have all previously demonstrated to play a role in TNBC chemoresistance, and many of these genes are regulated and stabilized by PIN1 (24). Therefore PIN1 loss could restore chemo-response. In our studies, we observed PIN1 levels to be significantly lower in TNBC BEAUTY PDX tumors obtained from patients that exhibited taxane treatment response compared to TNBC PDX resistant to taxane therapy (Fig. 4E). We also found that increasing the endogenous levels of ATRA, either through overexpressing RDH10 (Fig. 3D, E) or ATRA supplementation (Fig. 6C) decreased PIN1 levels (Fig. 5, 6B) and increased sensitivity to taxane treatment in TNBC cells (Fig. 5, 6D).

While PIN1 expression appears to be important to chemo-sensitivity in TNBC, we also showed PIN1 catalytic activity is equally important (Fig. 5). Work conducted by Lee et al. showed that Death-Associated Protein Kinase 1 (DAPK1) directly phosphorylates PIN1 at Ser71, rendering PIN1 catalytically inactive (26). DAPK1 is a well-known tumor suppressor often lost in cancer tissues (51-53); however high expression of DAPK1 only in the TNBC subtype is associated with greater relapse-free survival (Supplementary Fig. 4). Our cytotoxicity results with catalytically inactivate S71D PIN1 suggest that the phosphorylation status of Ser71 PIN1 in addition to PIN1 level is important for taxane sensitivity in TNBC. Future work should include confirming the role of DAPK1 in TNBC taxane sensitivity.

Therapeutic doses of ATRA, as seen in the treatment of APL, result in a peak plasma concentration of 1 uM (54). However, we only observed low PIN1 levels and improved taxane sensitivity at this concentration 
in BT549. At this concentration, RDH10 levels were found to be four times higher (Fig. 6B) resulting in three times greater intracellular ATRA concentrations (Fig. 6C) in BT549 compared to MDA-MB-231 cells. This suggests that ATRA-induced RDH10 expression could have contributed to intracellular ATRA concentrations to levels sufficient to degrade PIN1. Nevertheless, higher concentrations of ATRA resulted in increased taxane cytotoxicity for both BT549 and MDA-MB-231, regardless of RDH10 induction (Fig. 6D). Much effort is on-going to improve ATRA bioavailability including novel drug delivery mechanisms, such as ATRA-conjugated aptamers and nanoparticles (55). Furthermore in a phase I clinical trial, patients treated with liposomal ATRA (Atragen) achieved peak plasma concentrations of 25 UM (56). These efforts to improve ATRA bioavailability could benefit NAC treatment response for TNBC patients. Another reason contributing to the differences in ATRA levels between cell lines could be due to differences in cell line metabolism such as ATRA-dependent upregulation of CYPs, reducing the intracellular concentrations of ATRA. Interestingly, we observed that overexpression of RDH10 in-vitro had a significant down-regulation in CYP26A1 and CYP26B1 mRNA expression (Fig. 3F), compared with exogenous ATRA treatment (22). Therefore we hypothesize patients with RDH10 amplification not only increase endogenous levels of ATRA, but also inhibit its intracellular metabolism. This finding not only provides a potential rationale for TNBC patient response, but also supports a growing body of evidence of CYP expression-regulating genes that can significantly influence response to drug therapy (57).

Furthermore, retrospective genomic analysis of TNBC patient tumors and correlation with MRI tumor response following NAC revealed that $100 \%$ of patients with $\mathrm{RDH} 10$ amplification achieved pathological complete response compared $44.1 \%$ with wild-type levels of RDH10 (Supplementary Table 3). This finding supports RDH10 amplification as a strong candidate biomarker for predicting TNBC tumor response to neoadjuvant chemotherapy, and should be validated with additional cohorts.

In conclusion, here we demonstrated the benefit of screening individual genes from CNAmps associated with PCR following chemotherapy in TNBC patients to identify biomarkers and to elucidate additional treatment strategies. Nonetheless, most CNAs are large, containing many genes, and their association with treatment outcome may not be well justified by gene-centric approaches $(58,59)$. For example, "passenger" genes may influence the magnitude of the "driver" gene's effect on drug-response $(60,61)$. Work conducted by Xue et al., demonstrated that simultaneous suppression of genes within chromosome $8 p$ appeared to have a synergistic effect in promoting tumor growth compared to the loss of individual genes (62), suggesting large-scale deletions could create phenotypes that may fundamentally differ from those arising from loss of a single gene. Therefore systematically studying large-scale copy number aberrations in tumors would be a next step to predicting tumor behavior and treatment response, especially in highly heterogeneous diseases such as TNBC. Future generation of cancer cell line models to determine the effect of CNAs will require methods of whole-chromosome manipulation.

\section{Conclusion}

Utilizing genomic data from TNBC patients treated with neoadjuvant chemotherapy (NAC) enrolled on the Mayo BEAUTY study, we demonstrated RDH10 and its downstream target, PIN1, as potential biomarkers 
of taxane response. Retrospective analysis of MRI results from the BEAUTY patients confirmed a significant association of RDH10 amplification with pathological complete response following NAC, supporting RDH10 as a NAC response biomarker in TNBC, warranting future validation in additional cohorts. Furthermore, we show that ATRA, the downstream metabolite of RDH10 and retinoid metabolism, can increase TNBC cells sensitivity to paclitaxel. While further in-vivo studies are necessary to support our findings, repurposing ATRA, a drug with proven efficacy in acute promyelocytic leukemia, should be further investigated in combination with NAC to improve TNBC tumor response.

\section{List Of Abbreviations}

RDH10- Retinol dehydrogenase 10

BEAUTY-Breast Cancer Genome Guided Therapy Study

ATRA- All-Trans Retinoic Acid

PIN1- Peptidyl-prolyl cis-trans isomerase NIMA-interacting 1

TNBC- triple-negative breast cancer

CNAmps- copy number amplifications

HPLC- high-pressure liquid chromatography

HER2- human epidermal growth factor receptor 2

ER- estrogen receptor

PR-progesterone receptor

CNAs- copy number alterations

pCR- pathological complete response

NGS- next-generation sequencing

WES- whole-exome sequencing

RNAseq- RNA-sequencing

PTX-paclitaxel

Epi- epirubicin

ROL- all-trans retinol 
CYP- cytochrome p450

PDX- patient-derived xenograft

NAC- neoadjuvant chemotherapy

APL- acute promyelocytic leukemia

DAPK1- death-associated protein kinase 1

\section{Declarations}

\section{Ethics approval and consent to participate}

Not applicable

Consent for publication

Not applicable

\section{Availability of data and materials}

The datasets used and/or analyzed during the current study are available from the corresponding author on reasonable request.

\section{Competing interests}

The authors declare that they have no competing interests

\section{Funding}

The study was supported by National Institute of Health T32 Pre-doctoral Training Grant under award number GM072474 and CA196648 L.W, Mayo Clinic Center for Individualized Medicine. We thank Mayo Clinic Breast Cancer Genome Guided Therapy Study (BEAUTY) and Breast SPORE study team for performing genomic analysis and supply of Patient Derived Xenografts for this study.

\section{Authors' contributions}

JMG and LW designed the study. JMG, LZ and JY performed the experiments. PTV, KRK, VJS, JCB, MPG, and LW provided feedback, contributed to the interpretation of the results and supervised this project. All authors read and approved the final version of the manuscript.

\section{Acknowledgements}

We would like to acknowledge all co-authors involved throughout this project, the National Institute of Health T32 Pre-doctoral Training Grant under award number GM072474 and CA196648 L.W, Mayo Clinic 
Center for Individualized Medicine for supporting this study. We thank Mayo Clinic Breast Cancer Genome Guided Therapy Study (BEAUTY) and Breast SPORE study team for performing genomic analysis and supply of Patient Derived Xenografts for this study.

\section{References}

1. Akram M, lqbal M, Daniyal M, Khan AU. Awareness and current knowledge of breast cancer. Biol Res. 2017;50(1):33.

2. Palma G, Frasci G, Chirico A, Esposito E, Siani C, Saturnino C, et al. Triple negative breast cancer: looking for the missing link between biology and treatments. Oncotarget. 2015;6(29):26560-74.

3. Hirshfield KM, Ganesan S. Triple-negative breast cancer: molecular subtypes and targeted therapy. Curr Opin Obstet Gynecol. 2014;26(1):34-40.

4. Liedtke C, Mazouni C, Hess KR, Andre F, Tordai A, Mejia JA, et al. Response to neoadjuvant therapy and long-term survival in patients with triple-negative breast cancer. J Clin Oncol. 2008;26(8):127581.

5. Kalimutho M, Parsons K, Mittal D, Lopez JA, Srihari S, Khanna KK. Targeted Therapies for TripleNegative Breast Cancer: Combating a Stubborn Disease. Trends Pharmacol Sci. 2015;36(12):822-46.

6. Shlien A, Malkin D. Copy number variations and cancer. Genome Med. 2009;1(6):62.

7. de Smith AJ, Walters RG, Froguel P, Blakemore AI. Human genes involved in copy number variation: mechanisms of origin, functional effects and implications for disease. Cytogenet Genome Res. 2008;123(1-4):17-26.

8. Kalari KR, Hebbring SJ, Chai HS, Li L, Kocher JP, Wang L, et al. Copy number variation and cytidine analogue cytotoxicity: a genome-wide association approach. BMC Genomics. 2010;11:357.

9. Kim D, Shin H, Song YS, Kim JH. Synergistic effect of different levels of genomic data for cancer clinical outcome prediction. J Biomed Inform. 2012;45(6):1191-8.

10. Stuart D, Sellers WR. Linking somatic genetic alterations in cancer to therapeutics. Curr Opin Cell Biol. 2009;21(2):304-10.

11. Kumar N, Cai H, von Mering $\mathrm{C}$, Baudis M. Specific genomic regions are differentially affected by copy number alterations across distinct cancer types, in aggregated cytogenetic data. PLoS One. 2012;7(8):e43689.

12. Goetz MP, Kalari KR, Suman VJ, Moyer AM, Yu J, Visscher DW, et al. Tumor Sequencing and PatientDerived Xenografts in the Neoadjuvant Treatment of Breast Cancer. J Natl Cancer Inst. 2017;109(7).

13. Williams SJ, Cvetkovic D, Hamilton TC. Vitamin A metabolism is impaired in human ovarian cancer. Gynecol Oncol. 2009;112(3):637-45.

14. Hoffman LM, Garcha K, Karamboulas K, Cowan MF, Drysdale LM, Horton WA, et al. BMP action in skeletogenesis involves attenuation of retinoid signaling. J Cell Biol. 2006;174(1):101-13.

15. Gyorffy B, Lanczky A, Eklund AC, Denkert C, Budczies J, Li Q, et al. An online survival analysis tool to rapidly assess the effect of 22,277 genes on breast cancer prognosis using microarray data of 1,809 
patients. Breast Cancer Res Treat. 2010;123(3):725-31.

16. Lundberg E, Fagerberg L, Klevebring D, Matic I, Geiger T, Cox J, et al. Defining the transcriptome and proteome in three functionally different human cell lines. Mol Syst Biol. 2010;6:450.

17. Uhlen $M$, Oksvold P, Fagerberg L, Lundberg $E$, Jonasson $K$, Forsberg $M$, et al. Towards a knowledgebased Human Protein Atlas. Nat Biotechnol. 2010;28(12):1248-50.

18. McCleland ML, Mesh K, Lorenzana E, Chopra VS, Segal E, Watanabe C, et al. CCAT1 is an enhancertemplated RNA that predicts BET sensitivity in colorectal cancer. J Clin Invest. 2016;126(2):639-52.

19. Liden M, Eriksson U. Understanding retinol metabolism: structure and function of retinol dehydrogenases. J Biol Chem. 2006;281(19):13001-4.

20. Yamamoto Y, Zolfaghari R, Ross AC. Regulation of CYP26 (cytochrome P450RAI) mRNA expression and retinoic acid metabolism by retinoids and dietary vitamin $A$ in liver of mice and rats. FASEB J. 2000;14(13):2119-27.

21. Ross AC, Zolfaghari R. Cytochrome P450s in the regulation of cellular retinoic acid metabolism. Annu Rev Nutr. 2011;31:65-87.

22. Topletz AR, Thatcher JE, Zelter A, Lutz JD, Tay S, Nelson WL, et al. Comparison of the function and expression of CYP26A1 and CYP26B1, the two retinoic acid hydroxylases. Biochem Pharmacol. 2012;83(1):149-63.

23. Nelson $\mathrm{CH}$, Buttrick BR, Isoherranen $\mathrm{N}$. Therapeutic potential of the inhibition of the retinoic acid hydroxylases CYP26A1 and CYP26B1 by xenobiotics. Curr Top Med Chem. 2013;13(12):1402-28.

24. Wei S, Kozono S, Kats L, Nechama M, Li W, Guarnerio J, et al. Active Pin1 is a key target of all-trans retinoic acid in acute promyelocytic leukemia and breast cancer. Nat Med. 2015;21(5):457-66.

25. Chen Y, Wu YR, Yang HY, Li XZ, Jie MM, Hu CJ, et al. Prolyl isomerase Pin1: a promoter of cancer and a target for therapy. Cell Death Dis. 2018;9(9):883.

26. Lee TH, Chen CH, Suizu F, Huang P, Schiene-Fischer C, Daum S, et al. Death-associated protein kinase 1 phosphorylates Pin1 and inhibits its prolyl isomerase activity and cellular function. Mol Cell. 2011;42(2):147-59.

27. Degos L, Dombret H, Chomienne C, Daniel MT, Miclea JM, Chastang C, et al. All-trans-retinoic acid as a differentiating agent in the treatment of acute promyelocytic leukemia. Blood. 1995;85(10):264353.

28. Wu L, Chaudhary SC, Atigadda VR, Belyaeva OV, Harville SR, Elmets CA, et al. Retinoid X Receptor Agonists Upregulate Genes Responsible for the Biosynthesis of All-Trans-Retinoic Acid in Human Epidermis. PLoS One. 2016;11(4):e0153556.

29. Parisotto M, Brodeur H, Bhat PV, Mader S. [Retinoid metabolism and cancer]. Med Sci (Paris). 2006;22(12):1101-6.

30. Kuznetsova ES, Zinovieva OL, Oparina NY, Prokofjeva MM, Spirin PV, Favorskaya IA, et al. [Abnormal expression of genes that regulate retinoid metabolism and signaling in non-small-cell lung cancer]. Mol Biol (Mosk). 2016;50(2):255-65. 
31. Triano EA, Slusher LB, Atkins TA, Beneski JT, Gestl SA, Zolfaghari R, et al. Class I alcohol dehydrogenase is highly expressed in normal human mammary epithelium but not in invasive breast cancer: implications for breast carcinogenesis. Cancer Res. 2003;63(12):3092-100.

32. Hayden LJ, Satre MA. Alterations in cellular retinol metabolism contribute to differential retinoid responsiveness in normal human mammary epithelial cells versus breast cancer cells. Breast Cancer Res Treat. 2002;72(2):95-105.

33. Jette C, Peterson PW, Sandoval IT, Manos EJ, Hadley E, Ireland CM, et al. The tumor suppressor adenomatous polyposis coli and caudal related homeodomain protein regulate expression of retinol dehydrogenase L. J Biol Chem. 2004;279(33):34397-405.

34. Pasquali D, Thaller C, Eichele G. Abnormal level of retinoic acid in prostate cancer tissues. J Clin Endocrinol Metab. 1996;81(6):2186-91.

35. Guo E, Wei H, Liao X, Xu Y, Li S, Zeng X. Prognostic value of alcohol dehydrogenase mRNA expression in gastric cancer. Oncol Lett. 2018;15(4):5505-16.

36. Resetkova E, Reis-Filho JS, Jain RK, Mehta R, Thorat MA, Nakshatri H, et al. Prognostic impact of ALDH1 in breast cancer: a story of stem cells and tumor microenvironment. Breast Cancer Res Treat. 2010;123(1):97-108.

37. Rustighi A, Zannini A, Campaner E, Ciani Y, Piazza S, Del Sal G. PIN1 in breast development and cancer: a clinical perspective. Cell Death Differ. 2017;24(2):200-11.

38. Kozono S, Lin YM, Seo HS, Pinch B, Lian X, Qiu C, et al. Arsenic targets Pin1 and cooperates with retinoic acid to inhibit cancer-driving pathways and tumor-initiating cells. Nat Commun. 2018;9(1):3069.

39. Fan Y, Dutta J, Gupta N, Fan G, Gelinas C. Regulation of programmed cell death by NF-kappaB and its role in tumorigenesis and therapy. Adv Exp Med Biol. 2008;615:223-50.

40. Montagut C, Tusquets I, Ferrer B, Corominas JM, Bellosillo B, Campas C, et al. Activation of nuclear factor-kappa $B$ is linked to resistance to neoadjuvant chemotherapy in breast cancer patients. Endocr Relat Cancer. 2006;13(2):607-16.

41. Zhang GN, Zhang YK, Wang YJ, Gupta P, Ashby CR, Jr., Alqahtani S, et al. Epidermal growth factor receptor (EGFR) inhibitor PD153035 reverses ABCG2-mediated multidrug resistance in non-small cell lung cancer: In vitro and in vivo. Cancer Lett. 2018;424:19-29.

42. Liao WS, Ho Y, Lin YW, Naveen Raj E, Liu KK, Chen C, et al. Targeting EGFR of triple-negative breast cancer enhances the therapeutic efficacy of paclitaxel- and cetuximab-conjugated nanodiamond nanocomposite. Acta Biomater. 2019;86:395-405.

43. Nechushtan H, Vainer G, Stainberg H, Salmon AY, Hamburger T, Peretz T. A phase $1 / 2$ of a combination of cetuximab and taxane for "triple negative" breast cancer patients. Breast. 2014;23(4):435-8.

44. Balko JM, Giltnane JM, Wang K, Schwarz LJ, Young CD, Cook RS, et al. Molecular profiling of the residual disease of triple-negative breast cancers after neoadjuvant chemotherapy identifies actionable therapeutic targets. Cancer Discov. 2014;4(2):232-45. 
45. Campbell KJ, Dhayade S, Ferrari N, Sims AH, Johnson E, Mason SM, et al. MCL-1 is a prognostic indicator and drug target in breast cancer. Cell Death Dis. 2018;9(2):19.

46. Wertz IE, Kusam S, Lam C, Okamoto T, Sandoval W, Anderson DJ, et al. Sensitivity to antitubulin chemotherapeutics is regulated by MCL1 and FBW7. Nature. 2011;471(7336):110-4.

47. Steelman LS, Navolanic PM, Sokolosky ML, Taylor JR, Lehmann BD, Chappell WH, et al. Suppression of PTEN function increases breast cancer chemotherapeutic drug resistance while conferring sensitivity to mTOR inhibitors. Oncogene. 2008;27(29):4086-95.

48. Harrison H, Farnie G, Howell SJ, Rock RE, Stylianou S, Brennan KR, et al. Regulation of breast cancer stem cell activity by signaling through the Notch4 receptor. Cancer Res. 2010;70(2):709-18.

49. Rustighi A, Zannini A, Tiberi L, Sommaggio R, Piazza S, Sorrentino G, et al. Prolyl-isomerase Pin1 controls normal and cancer stem cells of the breast. EMBO Mol Med. 2014;6(1):99-119.

50. Zang S, Chen F, Dai J, Guo D, Tse W, Qu X, et al. RNAi-mediated knockdown of Notch-1 leads to cell growth inhibition and enhanced chemosensitivity in human breast cancer. Oncol Rep. 2010;23(4):893-9.

51. Bialik S, Kimchi A. The death-associated protein kinases: structure, function, and beyond. Annu Rev Biochem. 2006;75:189-210.

52. Michie AM, McCaig AM, Nakagawa R, Vukovic M. Death-associated protein kinase (DAPK) and signal transduction: regulation in cancer. FEBS J. 2010;277(1):74-80.

53. Raval A, Tanner SM, Byrd JC, Angerman EB, Perko JD, Chen SS, et al. Downregulation of deathassociated protein kinase 1 (DAPK1) in chronic lymphocytic leukemia. Cell. 2007;129(5):879-90.

54. Adamson PC. All-Trans-Retinoic Acid Pharmacology and Its Impact on the Treatment of Acute Promyelocytic Leukemia. Oncologist. 1996;1(5):305-14.

55. Gui K, Zhang X, Chen F, Ge Z, Zhang S, Qi X, et al. Lipid-polymer nanoparticles with CD133 aptamers for targeted delivery of all-trans retinoic acid to osteosarcoma initiating cells. Biomed Pharmacother. 2019;111:751-64.

56. Ozpolat B, Lopez-Berestein G, Adamson P, Fu CJ, Williams AH. Pharmacokinetics of intravenously administered liposomal all-trans-retinoic acid (ATRA) and orally administered ATRA in healthy volunteers. J Pharm Pharm Sci. 2003;6(2):292-301.

57. Qin S, Liu D, Kohli M, Wang L, Vedell PT, Hillman DW, et al. TSPYL Family Regulates CYP17A1 and CYP3A4 Expression: Potential Mechanism Contributing to Abiraterone Response in Metastatic Castration-Resistant Prostate Cancer. Clin Pharmacol Ther. 2018;104(1):201-10.

58. Beroukhim R, Mermel CH, Porter D, Wei G, Raychaudhuri S, Donovan J, et al. The landscape of somatic copy-number alteration across human cancers. Nature. 2010;463(7283):899-905.

59. Zack TI, Schumacher SE, Carter SL, Cherniack AD, Saksena G, Tabak B, et al. Pan-cancer patterns of somatic copy number alteration. Nat Genet. 2013;45(10):1134-40.

60. Cai Y, Crowther J, Pastor T, Abbasi Asbagh L, Baietti MF, De Troyer M, et al. Loss of Chromosome 8p Governs Tumor Progression and Drug Response by Altering Lipid Metabolism. Cancer Cell. 
2016;29(5):751-66.

61. Liu Y, Chen C, Xu Z, Scuoppo C, Rillahan CD, Gao J, et al. Deletions linked to TP53 loss drive cancer through p53-independent mechanisms. Nature. 2016;531(7595):471-5.

62. Xue W, Kitzing T, Roessler S, Zuber J, Krasnitz A, Schultz N, et al. A cluster of cooperating tumorsuppressor gene candidates in chromosomal deletions. Proc Natl Acad Sci U S A. 2012;109(21):8212-7.

\section{Figures}


A
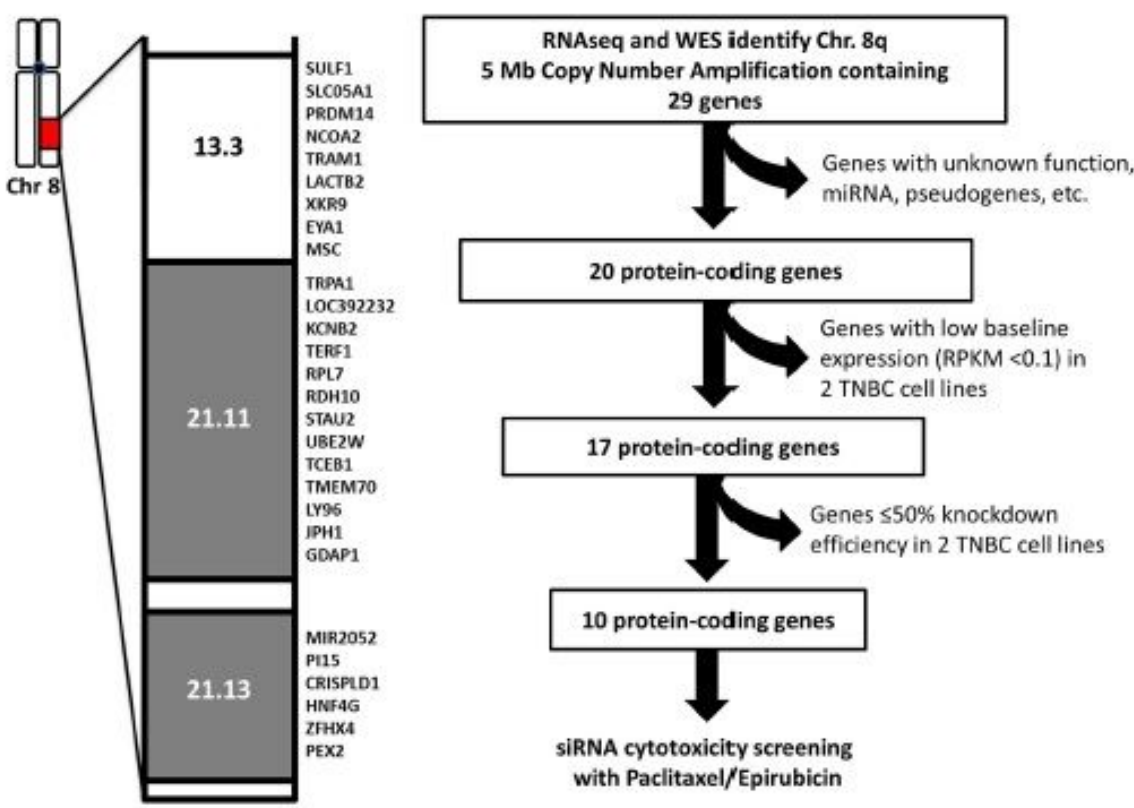

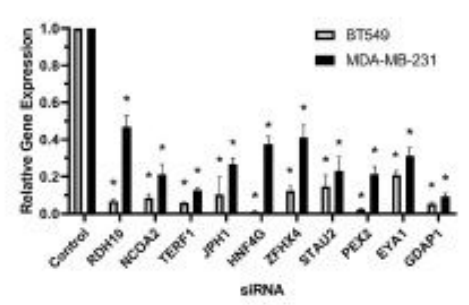

BT549

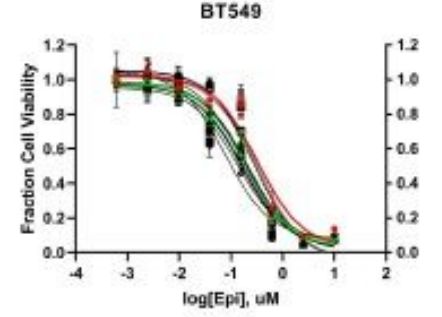

C

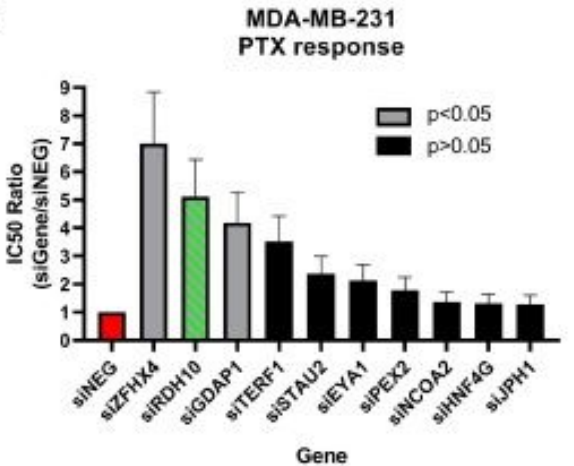

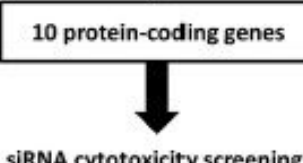

siRNA cytotoxicity screening with Paclitaxel/Epirubicin
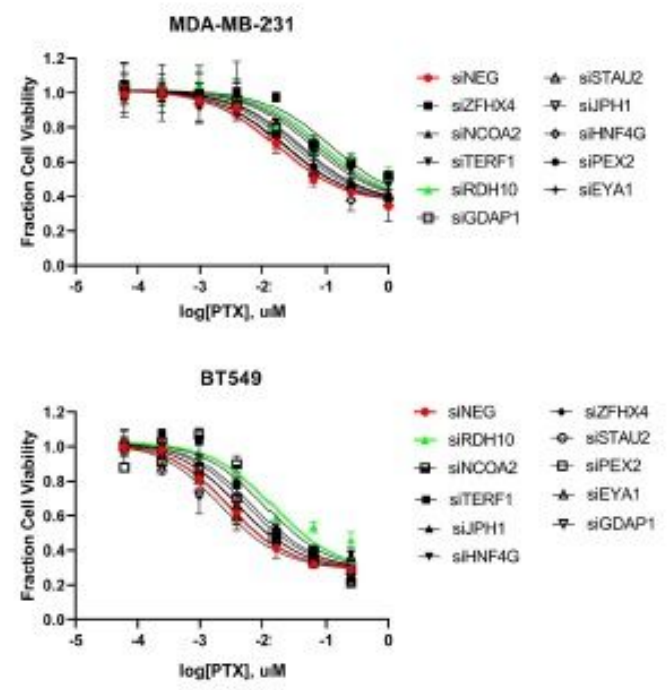

BT549

PTX response

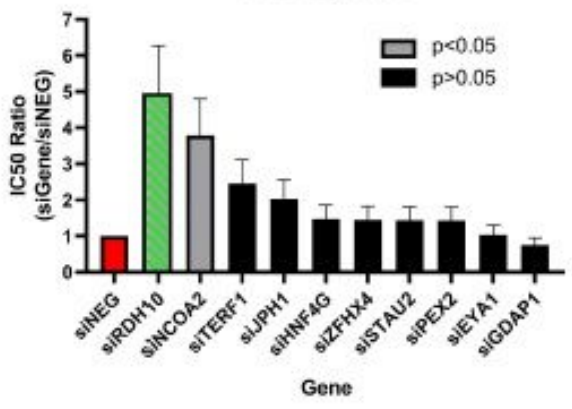

Figure 1

Gene selection and cytotoxicity screening of candidate genes in TNBC cell lines. (A) First, all genes with no-known-function, pseudogenes, and non-protein coding genes (e.g. IncRNA, miRNA, etc.) were removed from the 29 genes identified within the $5 \mathrm{Mb}$ copy number amplification identified in BEAUTY. Next, RNA sequencing data of available cell lines were utilized to select the two TNBC cell lines that expressed the majority of the 20 protein-coding genes. Any genes with low baseline cell line expression (RPKM< 0.1$)(16-$ 
18) were removed from our list, leaving 17 genes. Genes with $\leq 50 \%$ knockdown efficiency determined by qRT-PCR were removed, leaving 10 genes. (B) These genes were knocked down using siRNA in TNBC cell lines (mean +/-SD; $\left.{ }^{P}<0.001\right)$ followed by treatment with increasing concentrations of epirubicin or paclitaxel in BT549 and MDA-MB-231. (C) IC50 ratio comparing IC50 in cells with specific siRNAs targeting genes of interest to siNEG control for paclitaxel response. P-value was calculated using student t-test; Grey bars represent $\mathrm{P}<0.05$; black bars represent $\mathrm{P}>0.05$.

A

RDH10

Vinculin

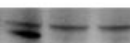

$=-$

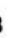

B
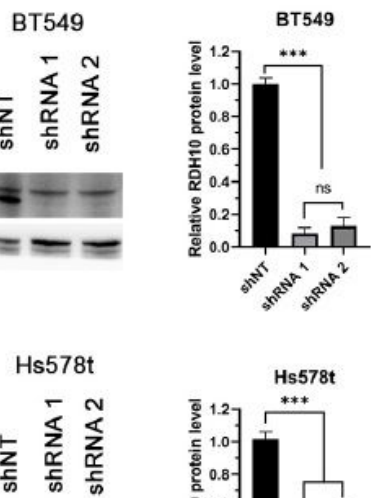

RDH10

Vinculin

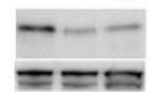

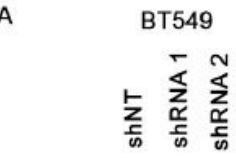
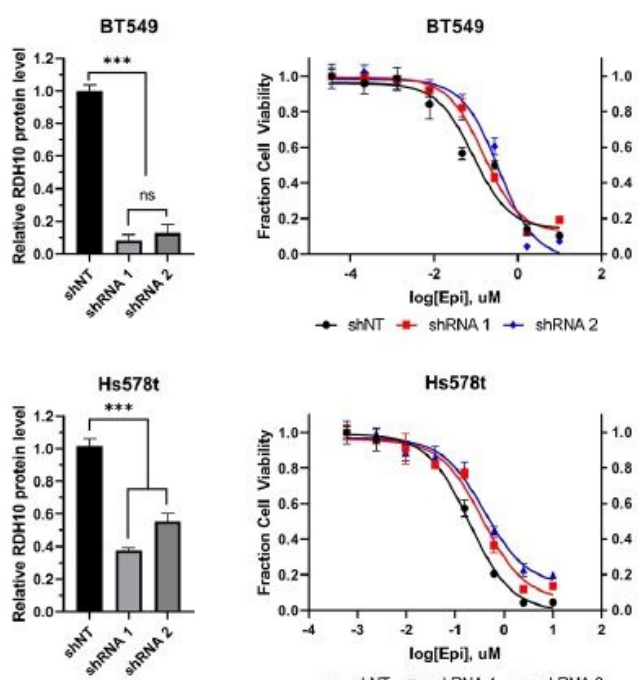

- shNT + shRNA1 1 shRNA2

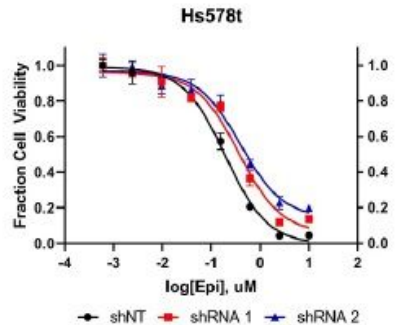

C

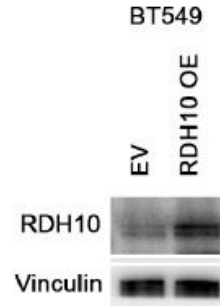

D

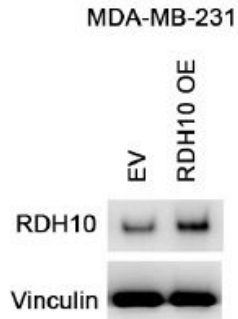

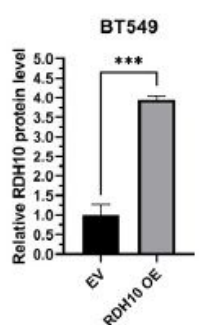

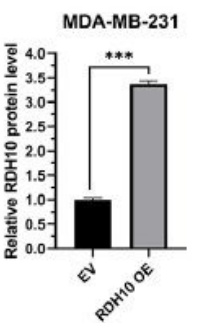

BT549

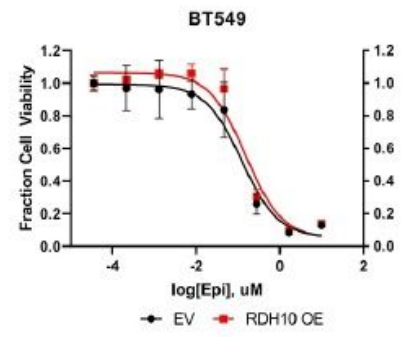

MDA-MB-231

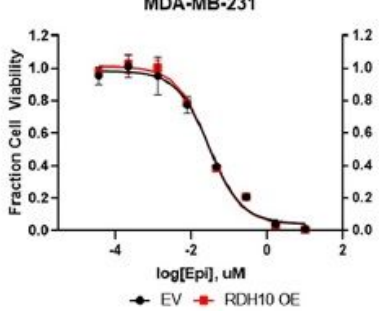

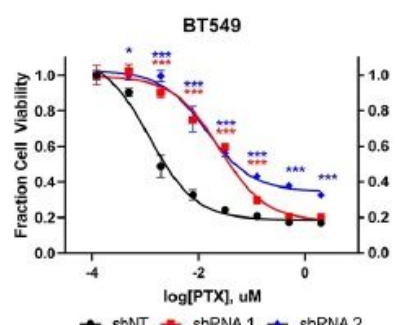

Hs578t

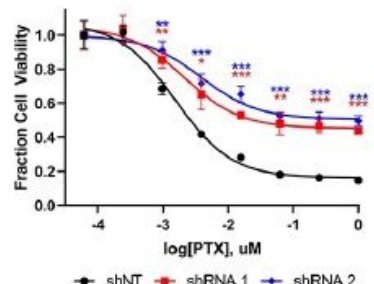

BT549

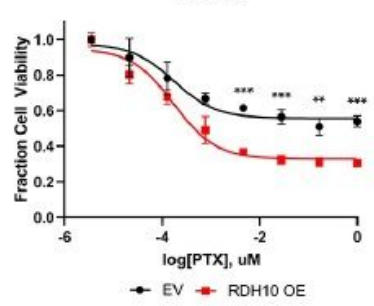

MDA-MB-231

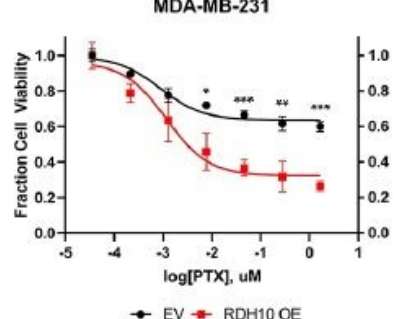

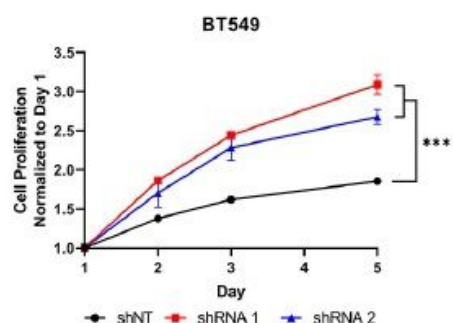

Hs578t

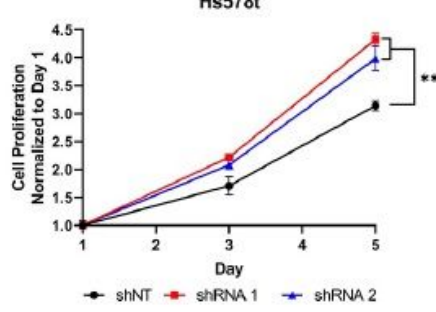

BT549

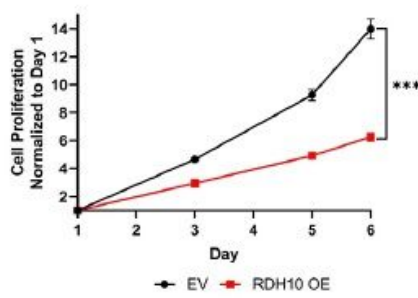

MDA-MB-231

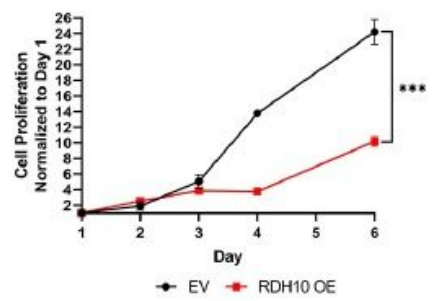

Figure 2

RDH10 expression affects cell proliferation and paclitaxel drug response in TNBC cell lines. Western blot for RDH10, epirubicin and paclitaxel dose-response curves and cell proliferation for RDH10 stable knockdown cell line using two shRNA in cell lines (A) BT549 and (B) Hs578t. Western blot for RDH10, epirubicin and paclitaxel dose-response curves, and cell proliferation for RDH10 overexpression in cell lines (C) BT549 and (D) MDA-MB-231. All quantifications normalized to vinculin and shNT or EV from $\mathrm{n}=3$ independent experiments. Cytotoxicity and cell proliferation experiments performed in triplicate. Data represent mean +/- SD. ${ }^{*} \mathrm{P}<0.01 ;{ }^{* \star \mathrm{P}}<0.001 ; * \star * \mathrm{P}<0.0001$, 2-tailed student t-test comparing individual shRNA or RDH10 OE to shNT or EV, respectively. 
A

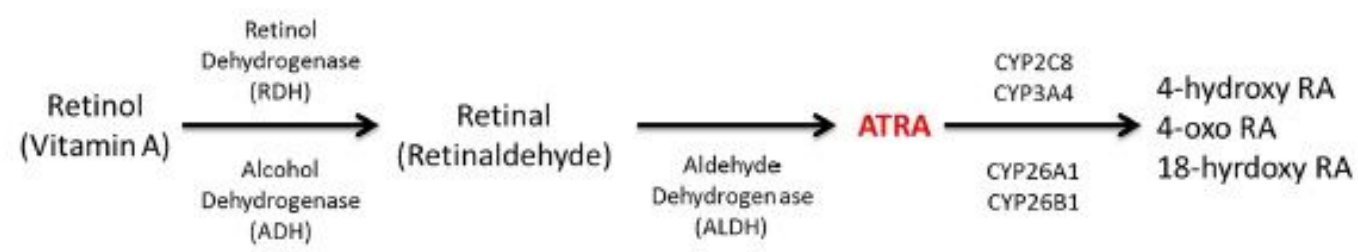

B
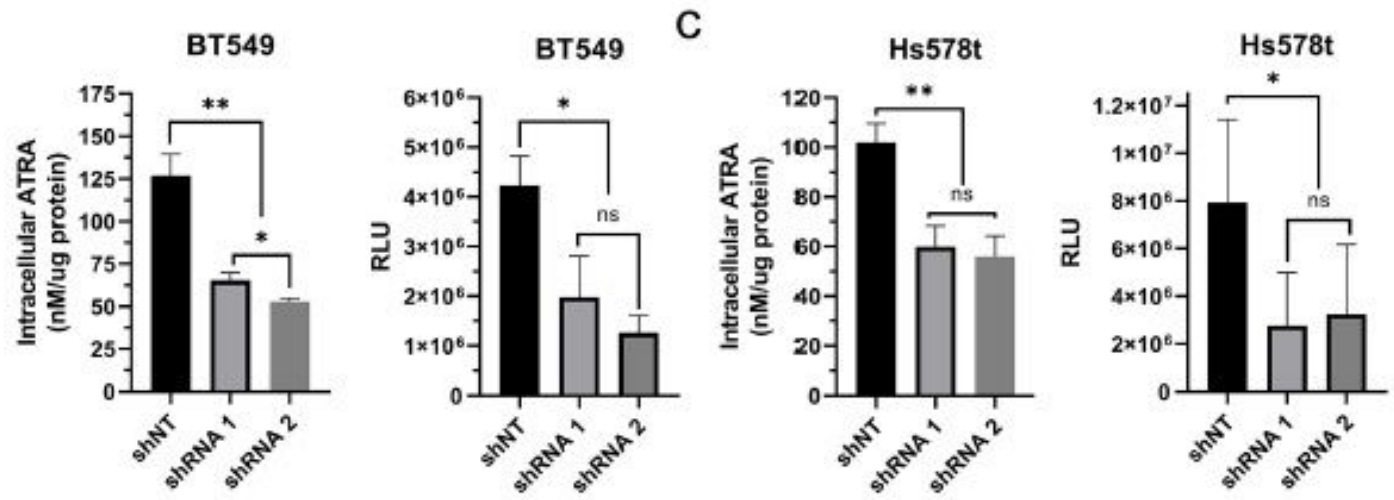

D
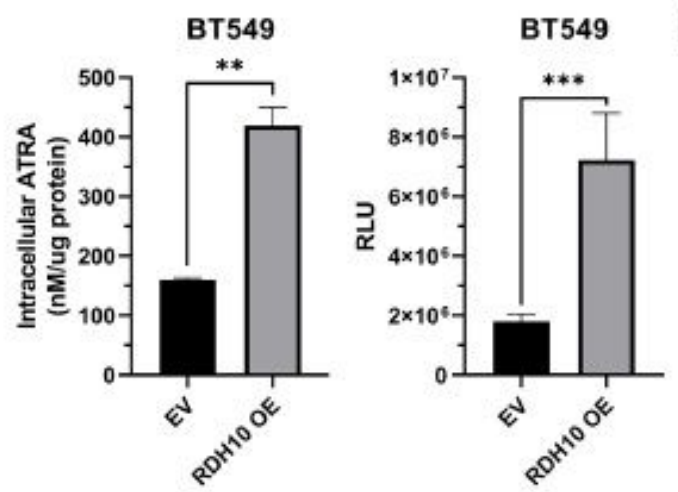

E

MDA-MB-231

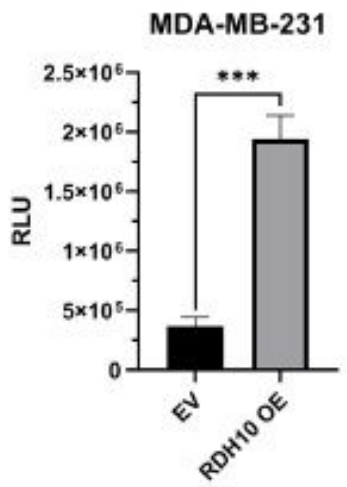

$\mathrm{F}$

BT549

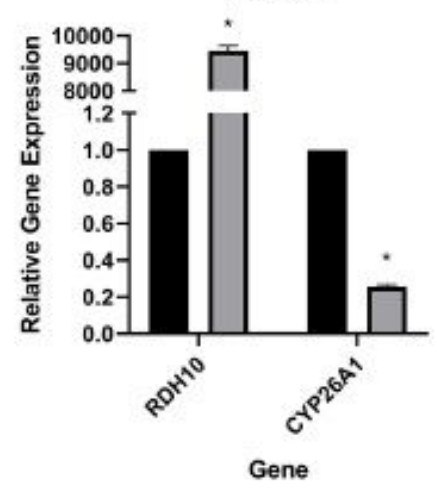

MDA-MB-231

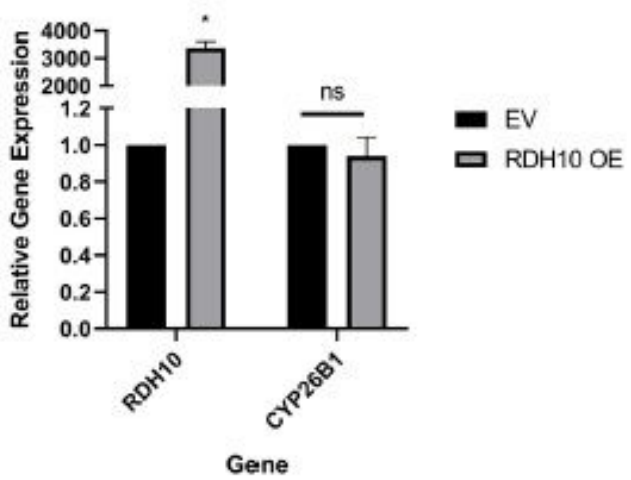

Figure 3

RDH10 expression affects intracellular ATRA concentration in TNBC cell lines. (A) Retinoid metabolism pathway. RDH10 is the rate-limiting step in the ATRA synthesis pathway. Measurement of intracellular ATRA concentration as determined by HPLC-UV detection using whole cell lysates and RARE-luciferase reporter assay in (B) BT549 RDH10 stable knockdown cell line, (C) Hs578t RDH10 stable knockdown cell line, (D) BT549 RDH10 overexpression cell line, and (E) MDA-MB-231 RDH10 overexpression cell line. (F) 
qRT-PCR of CYP26A1 and CYP26B1 in BT549 and MDA-MB-231 RDH10 overexpression cell lines. All experiments performed in quadruplicate. Data represent mean $+/$ - SD. ${ }^{*} P<0.01$; ${ }^{\star \star} P<0.001$; ${ }^{\star \star \star} P<0.0001$, 2-tailed student t-test.
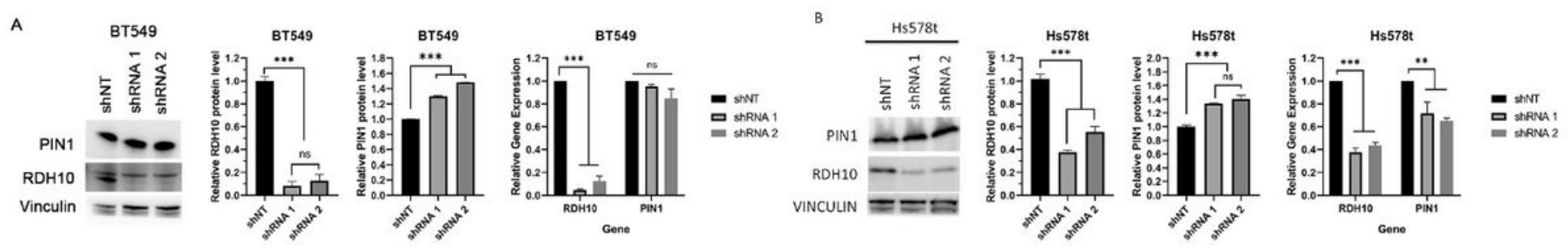

c
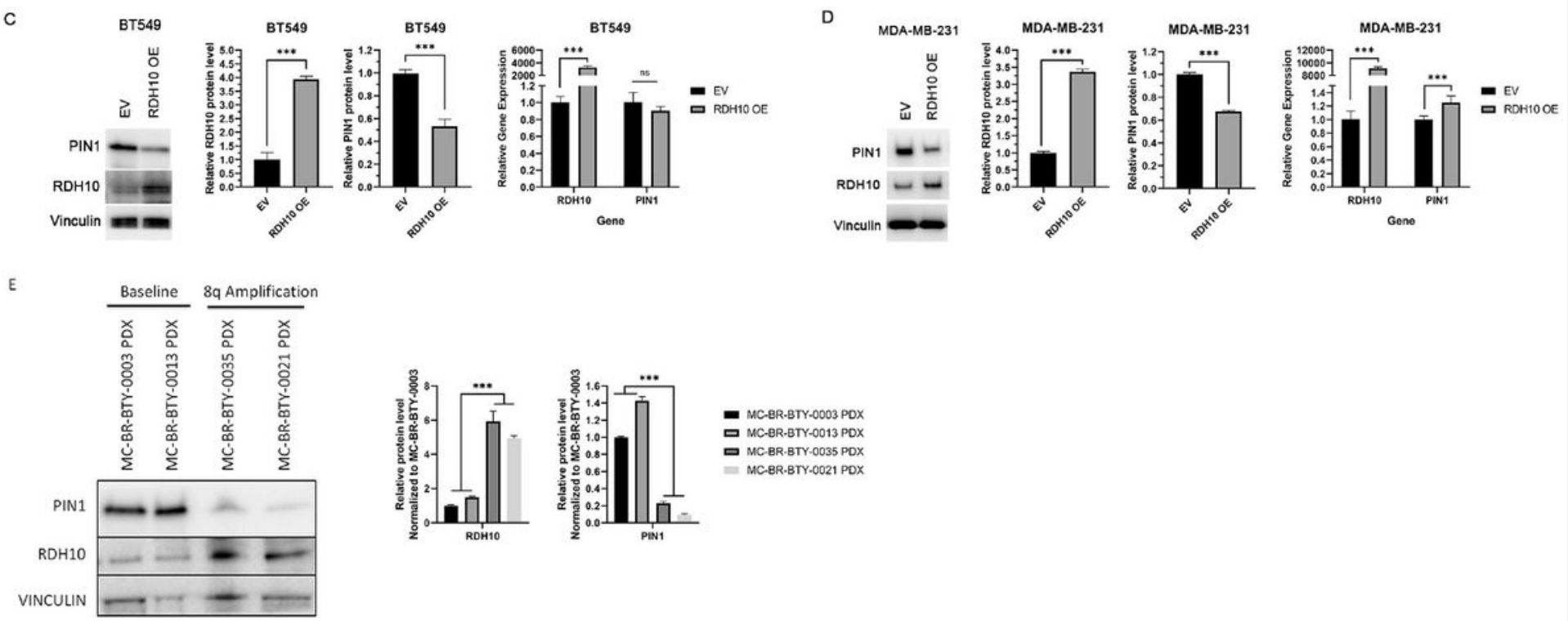

\section{Figure 4}

RDH10 expression inversely correlates with PIN1 protein expression in TNBC cell lines and BEAUTY PDX. Western blots and gene expression of RDH10 and PIN1 in (A) BT549 RDH10 stable knockdown cell line, (B) Hs578t RDH10 stable knockdown cell line, (C) BT549 RDH10 overexpression cell line, and (D) MDAMB-231 RDH10 overexpression cell line. (E) Western blot of RDH10 and PIN1 using tumor lysates of PDX with and without $8 \mathrm{q}$ amplification enrolled in BEAUTY. Quantification of western blots and qRT-PCR normalized to Vinculin or GAPDH then shNT or EV from $n=3$ independent measurements. Data represent mean $+/$ - SD. ${ }^{*} P<0.01 ;{ }^{*} \mathrm{P}<0.001 ; * \star * P<0.0001$, 2-tailed student t-test. 
A

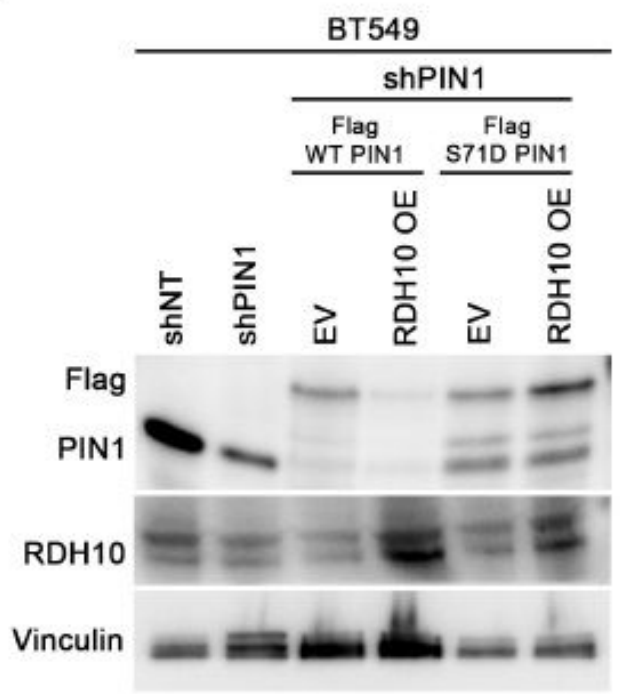

B

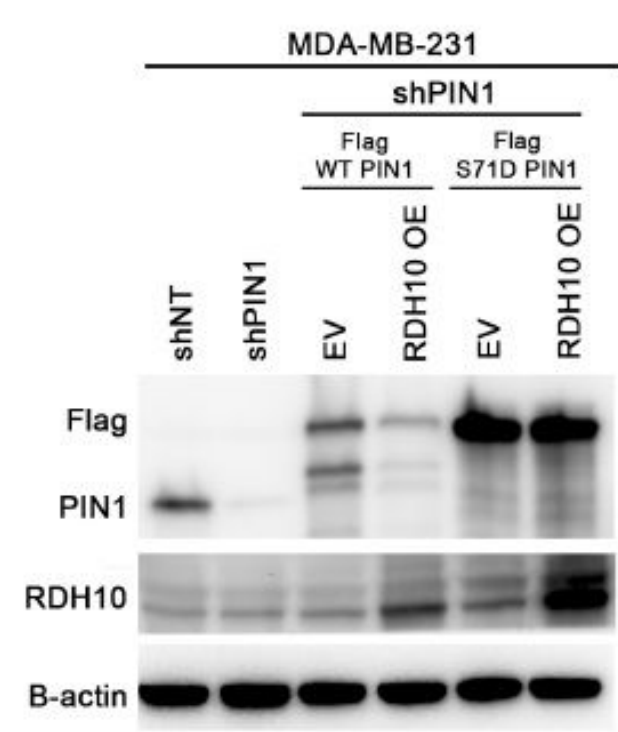

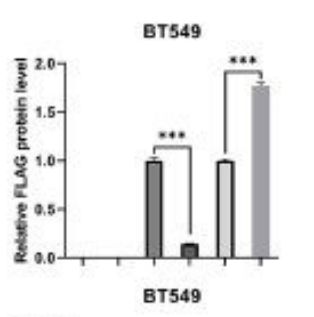
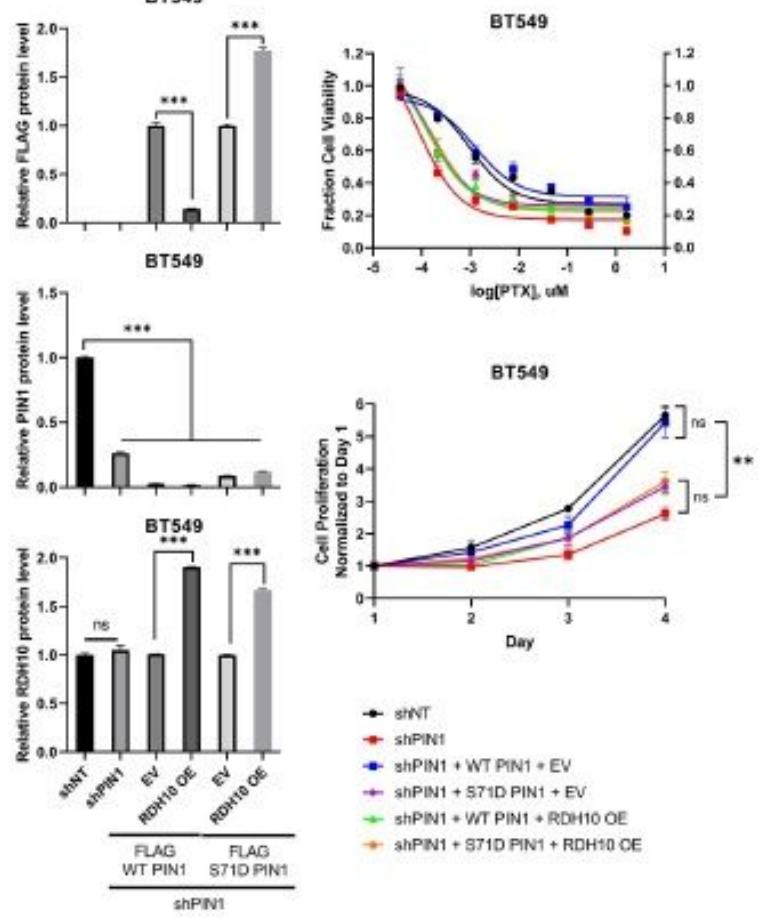

$\rightarrow$ stwT

- shipini

- shPIN1 + WT PIN1 + EV

- shPIN1 +\$71D PIN1 + EV

- ShPN1 + WT PIN1 + RDH10 OE

+ shPN1 + S71D PIN1 + RDH10 CE
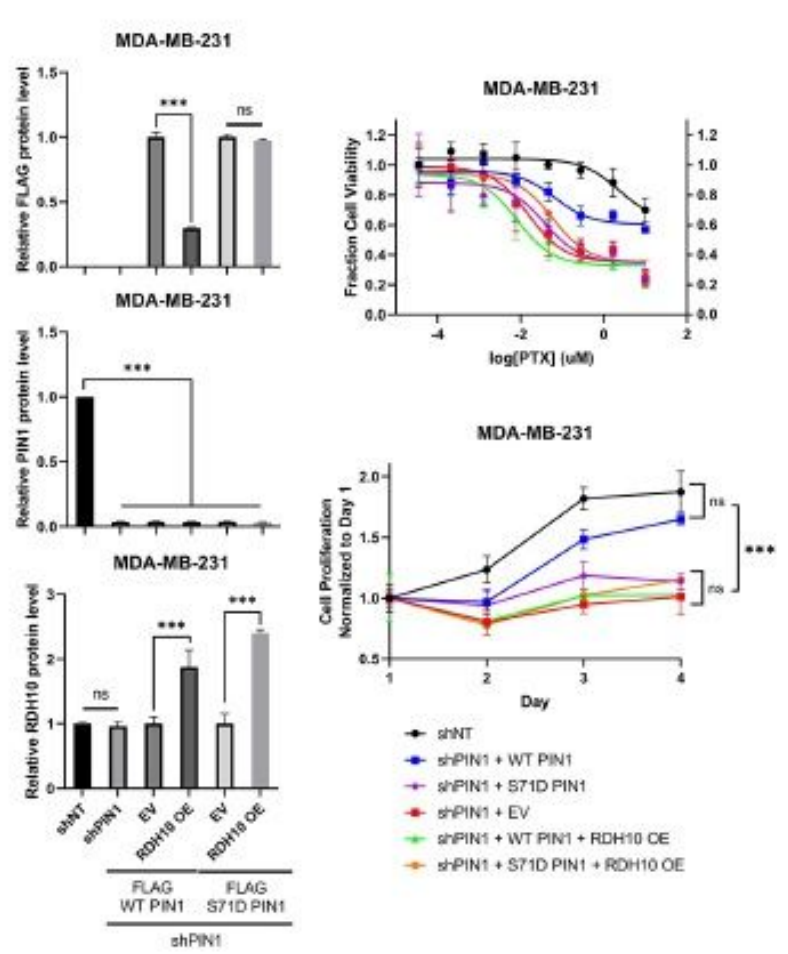

$\rightarrow$ giNT

- sPIN1 + WT PINT

- SFIN1 + ST1D FIN1

- STPIN1 + EV

- gRPIN1 + WT PIN1 + RDH10 CE

- SPIN1 + ST1DPIN1 + ROH10 OE

\section{Figure 5}

PIN1 is a downstream target of RDH10. Western blot, cell proliferation and paclitaxel cytotoxicity of (A) MDA-MB-231 and (B) BT549 PIN1 stable knockdown cell lines transfected with flag-tagged wild-type PIN1, flag-tagged catalytically inactive S71D PIN1, with EV or RDH10 overexpression constructs. Cell proliferation and cytotoxicity assays performed in triplicate. Quantification of western blots normalized to vinculin or b-actin and shNT or EV from $n=3$ independent measurements. Data represent mean +/- SD. *P 
$<0.01 ;{ }^{* \star} \mathrm{P}<0.001 ;{ }^{* \star \star} \mathrm{P}<0.0001,2$-tailed student t-test. Statistical comparisons for cytotoxicity curves see Supplementary Table 2.

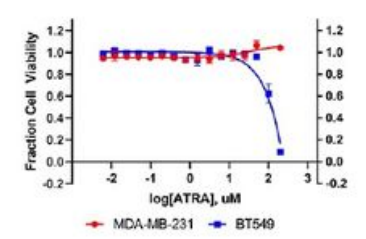

C

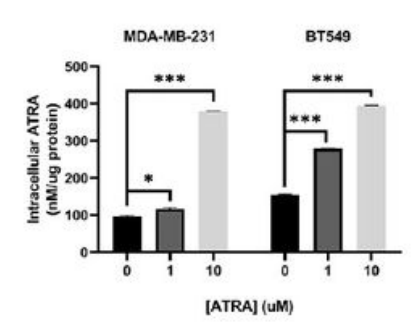

B
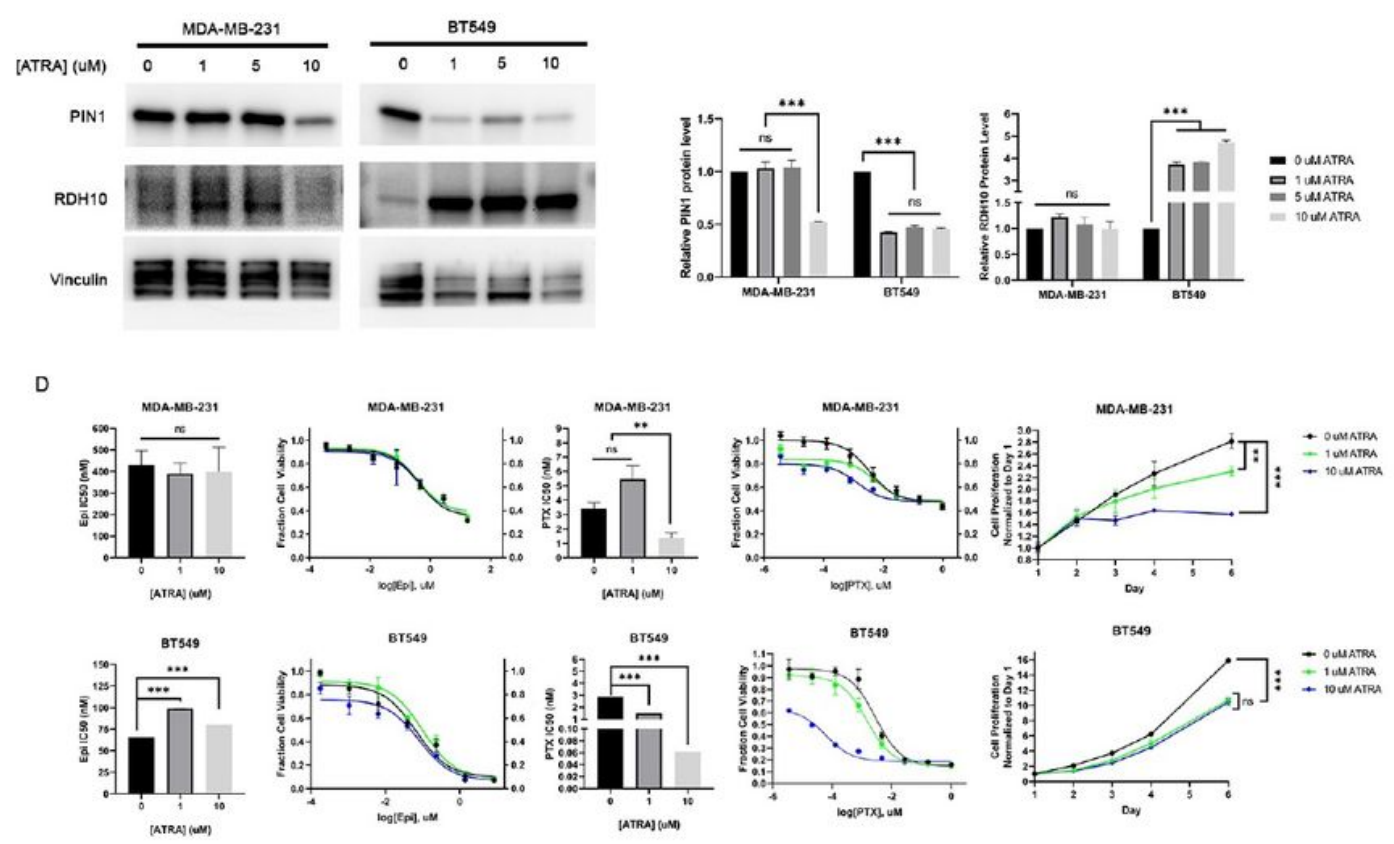

Figure 6

ATRA decreases PIN1, increases chemo-sensitivity and decreases cell proliferation in two TNBC cell lines. (A) ATRA dose-response in TNBC cell lines MDA-MB-231 and BT549. (B) Western blot of PIN1 and RDH10 in MDA-MB-231 and BT549 after treating with EtOH, 1 uM, 5 uM, or 10 uM ATRA for 72hr. (C) Intracellular ATRA concentrations of ATRA treated MDA-MB-231 and BT549 cells treated for 72 hours. (D) Cell cytotoxicity, IC50 comparisons and cell proliferation of MDA-MB-231 and BT549 cells treated with indicated concentrations of ATRA and paclitaxel or epirubicin. Cell proliferation and cytotoxicity assays performed in triplicate. Data represent mean +/- SD. ${ }^{*} \mathrm{P}<0.01$; ${ }^{*} \mathrm{P}<0.001$; $* \star * \mathrm{P}<0.0001$, 2-tailed student ttest.

\section{Supplementary Files}

This is a list of supplementary files associated with this preprint. Click to download.

- SupplementaryTable1.xlsx

- SupplementaryTable2.xlsx

- SupplementaryTable3.xlsx

- SUPPLEMENTARYFIGURE1..pdf

- SUPPLEMENTARYFIGURE2..pdf

- SUPPLEMENTARYFIGURE3..pdf

- SUPPLEMENTARYFIGURE4.pdf 